\title{
In-house night call management of four ectopic pregnancies
}

\begin{abstract}
Background: During the first trimester of pregnancy, ectopic pregnancy is the leading cause of maternal mortality. Diagnosis and management of ectopic pregnancies require that health care providers maintain vigilance. Providing a variety of surgical and medical treatments at night can be challenging to the on call team in a teaching hospital. We present four cases of ectopic pregnancies managed over a 15 hour period by the night on call gynecology team.

Cases: Case 1, 26-year-old women diagnosed with a ruptured ectopic pregnancy that required emergent laparotomy and blood transfusion. In Case 2, 27-year-old women underwent dilation, curettage and minimal invasive surgery of operative laparoscopy for ruptured ectopic pregnancy. In Case 3, a 24-year-old woman with unruptured ectopic pregnancy was treated with methotrexate. In Case 4, 35 year-old women underwent minimal invasive operative laparoscopy for unruptured ectopic pregnancy.

Conclusion: Despite technological advances in early diagnosis of ectopic pregnancy with the use of transvaginal sonogram and serial $\beta$-HCG titers, patients still present to the emergency department with ruptured ectopic pregnancy necessitating acute surgical interventions. This is especially prevalent in inner city hospital catchment area where patients often wait until development of severe symptoms before presenting for medical care. Therefore, diagnosis of ectopic pregnancies warrens a high index of suspicion and treatment plans that are individualized, particularly to area of services with attention to patient compliance.
\end{abstract}

Keywords: ectopic pregnancy, methotrexate (MTX), salpingectomy, transvaginal ultrasound (TVUS)
Volume 4 Issue 2 - 2016

\author{
Takeko Takeshige,' Shadi Rezai,' Thomas \\ Bemis, ${ }^{2}$ Annika Chadee, ' Viviene Bailey- \\ Gayle,' Umeko Takeshige, ${ }^{3}$ Ray Mercado,' \\ Cassandra E Henderson' \\ 'Department of Obstetrics and Gynecology,Weill Medical \\ College of Cornell University, USA \\ ${ }^{2}$ St.George's University, West Indies \\ ${ }^{3} \mathrm{New}$ York Institute of Technology College of Osteopathic \\ Medicine, USA
}

Correspondence: Takeko Takeshige, Department of Obstetrics and Gynecology, Lincoln Medical and Mental Health Center, 234 East 149th Street, Bronx, New York, USA Email takekolI@yahoo.com

Received: December 16, 2015 | Published: January 29, 2016

\section{Introduction}

During the first trimester of pregnancy, ectopic pregnancy is the leading cause of maternal mortality. Diagnosis and management of ectopic pregnancies require high vigilance by the provider. Ectopic pregnancy occurs when the developing Blastocyst becomes implanted at a site other than the endometrium of the uterine cavity. The most common extra-uterine location is the fallopian tube, which accounts for 98 percent of all ectopic gestations. Conservative approaches that attempt to save the fallopian tube are now first line before salpingectomy. However, hemorrhage from ectopic pregnancy is still the leading cause of pregnancy related maternal death in the first trimester despite improved diagnostic methods leading to earlier detection and treatment. ${ }^{1}$ Understanding the different treatments of ectopic pregnancy remains important in determining a treatment course, particularly in high volume, diverse patient populations.

We are part of the New York City Health + Hospitals network where the majority of patients do not present to health care facilities until they are very ill. The majority of patients in inner city areas is immigrants from developing countries and are considered at higher risks for development of sexually transmitted disease. ${ }^{2}$ Due to adhesions and other consequences associated with STDs, patients are at higher risk for ectopic pregnancy after STDs. Additionally, patients are often do not adhere to medical advice, making the implementation of appropriate treatment challenging. In this report, we describe four cases of ectopic pregnancies diagnosed in the emergency department and successfully managed either medically or surgically by the on-call gynecologic team over a 15 hour period.

\section{Four case series}

Presentation of the case 1: A 26-year-old women, gravida 4, para 3, last menstrual period of 4 weeks ago, with history of cesarean sections $\mathrm{x} 3$ and tubal ligation presented to the emergency department with acute onset of lower abdominal pain and vaginal bleeding. The urine pregnancy test was positive. On examination, abdomen was soft but diffusely tender with rebound and guarding. Scanty blood was noted in the vaginal vault. Uterus was normal in size. Bilateral adnexa were diffusely tender. Bedside transvaginal sonogram revealed no intrauterine pregnancy, large amount of fluid in the cul-de-sac, and $5 \mathrm{~cm}$ complex mass on the right adnexa. Ruptured ectopic pregnancy was diagnosed and patient taken to operating room for exploratory laparotomy.

Intraoperative findings of $1000 \mathrm{~mL}$ of hemoperitoneum, multiple dense adhesions, bilateral partial salpingectomy and ruptured left tubal pregnancy at ampulla were noted. Lysis of adhesions and partial left salpingectomy were performed. Patient was then transfused intraoperatively with three units of packed red blood cells due to tachycardia and hypotension. Her postoperative course was uneventful.

Presentation of the case 2: A 27 year-old female, gravida 3, para 1, with last menstrual period of 12 weeks ago presented to emergency department with complaints of vaginal bleeding and right lower quadrant pain. Her urine pregnancy test was positive.

On examination, abdomen was soft, but minimally tender on right lower quadrant without guarding and rebound. Scanty blood in the 
vaginal vault, normal sized uterus and mild right lower quadrant pain and tenderness were noted on pelvic examination. Bedside transvaginal sonogram revealed no intrauterine pregnancy, complex adnexal mass and presence of minimal fluid in the cul-de-sac. The serum HCG level was $9,347 \mathrm{mIU} / \mathrm{mL}$ and hemoglobin was $10.8 \mathrm{~g} / \mathrm{dL}$. Differential diagnoses were complete abortion with ruptured cyst and ectopic pregnancy. The patient was taken to the operation room for minimal invasive surgery of dilatation, curettage, and laparoscopy. Specimen obtained from curettage did not float, so laparoscopy was performed. Hemoperitoneum of $200 \mathrm{CC}$, dense pelvic side wall adhesions, hydrosalpinx, clubbed-ended tubes and ruptured right oviduct were noted at ampulla. Lysis of adhesions and right salpingectomy was performed. The patient had uneventful post op course.

Presentation of the case 3: A 24 year-old female, gravida 3, para 1 , with last regular menstrual period of 6 weeks ago, presented with vaginal bleeding and cramps. The urine pregnancy test was positive. On examination, abdomen was soft with minimal tenderness on right lower quadrant, but no guarding or rebound tenderness noted. Vaginal vault with scanty blood, cervical os closed, normal size uterus and nontender adnexa were palpated. Hemoglobin and serum HCG levels were $11.6 \mathrm{~g} / \mathrm{dL}$ and $1,109 \mathrm{mIU} / \mathrm{mL}$, respectively. Beside transvaginal ultrasound indicated no intrauterine pregnancy with complex left adnexal mass and small amount of fluid in the cul-de-sac. Patient strongly desired the pregnancy and was admitted to gynecology ward for observation with repeat $\beta$-HCG.

In spite of resolution of pain and vaginal bleeding, her serum HCG level increased to $1582 \mathrm{mIU} / \mathrm{mL}$. Patient opted for methotrexate. The patient failed to keep appointment on day 4 for $\beta$-HCG. Instead, she went to another hospital where laparoscopic salpingectomy was performed.

Presentation of the case 4: A 35 year-old female, gravida 10, para 6, last menstruation was 10 weeks ago presented with vaginal bleeding. The urine pregnancy test was positive. On examinations: abdomen was soft, nontender, no guarding and rebound; vagina: scanty blood; uterus normal in size and no adnexal tenderness or palpable mass noted. Laboratory results indicated hemoglobin of $11.6 \mathrm{~g} / \mathrm{dL}$ and serum HCG level of $33,296 \mathrm{mIU} / \mathrm{mL}$. Bedside transvaginal sonogram demonstrated no intrauterine pregnancy, right adnexal mass with gestational sac, yolk sac, and $3 \mathrm{~mm}$ embryo with cardiac activity of $100 \mathrm{bpm}$.

The patient was counseled and advised to undergo a laparoscopy for unruptured ectopic pregnancy, but declined surgery and left against medical advice. Later on, the patient returned for minimal invasive surgery, undergoing operative laparoscopy with right partial salpingectomy. The patient had an uncomplicated postoperative course.

\section{Discussion}

Ectopic pregnancy is the leading cause of maternal mortality in the first trimester of pregnancy. The incidence of ectopic pregnancy has plateaued at about 20 per 1,000 pregnancies in the early 1990 s, according to the last time the CDC reported national data on ectopic pregnancy. ${ }^{2}$ This trend is also consistent with the incidence of pelvic inflammatory disease attributed to both chlamydia and gonorrhea as similar in our institution. ${ }^{3}$ Other risk factors such as prior surgeries, multiple sexual partners, and smoking are also common in our hospital population. The incidence of ectopic pregnancy currently is difficult to estimate due to the increase in outpatient management of ectopic pregnancy. ${ }^{4}$ Estimates show that the ectopic pregnancy maternal mortality ratio declined from 1.15 to 0.5 deaths per 100,000 births, and projections predict that the rate will further decline between 20132017. ${ }^{5}$ This downward trend can be attributed to increased prenatal care and clinical management of ectopic pregnancy.

Typical symptoms of ectopic pregnancy are vaginal bleeding and abdominal pain after delayed menstruation. However, they are not specific because they are also present in all entities of abortions, making early diagnosis difficult. ${ }^{6,7}$ These associated symptoms are also not consistent with stage of the disease progression at time of presentation for evaluation. Patients may present in the early phase of ectopic pregnancy with minimal symptoms due to levels of progesterone, estradiol, and beta-HCG being lower than in normal pregnancy. ${ }^{8-10}$ Such cases may be treated with either observation or methotrexate administration without invasive procedures. However, the majority of patients in our city hospital catchment area presented to the emergency department with advanced stage symptoms, which is often accompanied by fetal cardiac activity in the ectopic sac pending rupture. It is often too late for conservative treatment and patients will require emergency surgeries.

Although serial transvaginal sonogram and $\beta$-HCG have enabled clinicians to diagnose ectopic pregnancy, diagnoses can be challenging when $\beta$-HCG levels are below discriminatory zone of 1,500-2,500IU/ L. ${ }^{11}$ An increase of $\beta$-HCG more than $66 \%$ in 48 hours is seen in $85 \%$ of intrauterine viable pregnancy, but slow increase or decrease of $\beta$-HCG cannot discriminate ectopic pregnancy from spontaneous abortion.

Transvaginal Ultrasound (TVUS) is the most useful method of diagnosing ectopic pregnancy, with the most common finding being an adnexal mass. ${ }^{12,13}$ A finding of any non-cystic adnexal mass by transvaginal sonogram had sensitivity, specificity, positive predictive value, and negative predictive value of $91 \%, 100 \%, 94 \%$ and $100 \%$, respectively. ${ }^{14}$ If TVUS is non-diagnostic, the gestation may be too early to be visualized via ultrasound, in which case serial measurements of serum hCG concentration should be taken until the discriminatory zone is reached. ${ }^{15}$

While the vast majority of ectopic pregnancies occur in the ampulla, TVUS can also diagnose more rare forms of ectopic pregnancy. Findings such as an interstitial line sign are highly sensitive (80\%) and specific (98\%) for interstitial pregnancy. ${ }^{16}$ Use of color Doppler has also shown high sensitivity and specificity for diagnosing rare forms of cesarean section scar and ovarian ectopic pregnancies.

Recent literature suggests that uterine curettage should not be routinely used in diagnostic process of ectopic pregnancy ${ }^{17}$ However, clinicians should combine clinical evaluation with culdocentesis or uterine curettage when ultrasound and $\beta-\mathrm{HCG}$ are not clear for diagnosis. If curettage is performed, the $\beta-\mathrm{HCG}$ should drop by at least $15 \%$ per day. ${ }^{18}$

Patients with early diagnosis of ectopic pregnancy can be managed conservatively. Options include expectant management, medical treatment with MTX, ultrasound-guided local injection of MTX or potassium chloride (KCl).$^{19}$ Expectant management may be considered when $\beta$-HCG level is less than $1500 \mathrm{mIU} / \mathrm{mL}$, no evidence of rupture, and $\beta-\mathrm{HCG}$ declines within 48 hours. MTX administration is a single dose of $50 \mathrm{mg} / \mathrm{m}^{2}$ body surface area, however the presence of fetal cardiac activity and high $\beta-\mathrm{HCG}$ level (greater than $5000 \mathrm{mIU} /$ $\mathrm{mL}$ ) are considered relative contraindications. ${ }^{20}$ Above options are contraindicated, if patients are hemodynamically unstable or noncompliant for follow up as that of our population. The latter will require surgery, preferably laparoscopy as $96 \%$ of women with ectopic pregnancies were successfully managed by laparoscopic procedures. ${ }^{21}$ 
In our clinical setting, serial $\beta$-HCG measurements might not be feasible because of three reasons:

i. Patients do not present to hospital when menses are delayed or until they are very ill

ii. Ultrasound is not diagnostic if $\beta-\mathrm{HCG}$ level is less than the discriminatory zone, and

iii. Patient compliance for follow up is poor due to high mobilization rates. Thus, laparoscopy plays an important role for both diagnostic and therapeutic modality in our setting.

\section{Summary and conclusion}

We reported four cases over a 15 hour shift of ruptured and unruptured ectopic pregnancies over 15 hours shift in various diagnostic stages. Majority of our patients are not candidates for medical management due to inconsistent medical follow-up. Prompt and precise evaluations with medical or surgical management are essential components to optimize patient outcome. Health Care Providers in the emergency department as well as the gynecology on call team should be familiar with both the diagnostic and therapeutic modalities of ectopic pregnancy in order to expedite the safest patient care.

Also, because of this city hospital catchment area, the likelihood of early diagnosis of an ectopic pregnancy is low; therefore, education for the risk of sterilization failure must be reinforced preoperatively and postoperatively for tubal ligation particularly in younger patients. ${ }^{22}$ Patients should also be counseled about the early symptoms of an ectopic pregnancy so that they may present to the hospital before complications occur.

Despite technological advances in early diagnosis of ectopic pregnancy with the use of transvaginal sonogram and serial $\beta-\mathrm{HCG}$ titers, patients still present to the emergency department with ruptured ectopic pregnancy necessitating acute surgical interventions. This is especially prevalent in inner city hospital catchment area where patients often wait until development of severe symptoms before presenting for medical care. ${ }^{23,24}$ Therefore, diagnosis of ectopic pregnancies warrens a high index of suspicion and treatment plans that are individualized, particularly to area of services with attention to patient compliance. ${ }^{25,26}$

Prior tubal surgery with or without cesarean, has been demonstrated to increase the risk of ectopic pregnancy. ${ }^{27}$ Upon presentation to the ED with the typical symptoms in a woman of childbearing age, a brief history of lifestyle with clinician evaluation may detail the patient's risk of an ectopic pregnancy. Although the management for ectopic pregnancy is often conservative, the evaluation for surgical management due to ruptured ectopic pregnancy has to remain heightened. Therefore, the on call gynecological team must be vigilant for proper diagnosis and emergency surgery.

\section{Acknowledgments}

Special thanks to Ms. Judith Wilkinson, Medical Librarian, from Lincoln Medical and Mental Health Center Science Library for assistance in finding the reference articles.

\section{Conflicts of interest}

The authors declare there is no conflict of interests

\section{Funding}

None.

\section{References}

1. Murray $\mathrm{H}$, Baakdah $\mathrm{H}$, Bardell $\mathrm{T}$, et al. Diagnosis and treatment of ectopic pregnancy. CMAJ. 2005;173(8):905-912.

2. Centers for Disease Control and Prevention (CDC) Ectopic pregnancyUnited States, 1990-1992. MMWR Morb Mortal Wkly Rep 44(3):4648.

3. Kamwendo F, Forslin L, Bodin L, et al. Epidemiology of ectopic pregnancy during a 28 year period and the role of pelvic inflammatory disease. Sex Transm Infect. 2000;76(1):28-32.

4. Zane SB, Kieke BA Jr, Kendrick JS, et al. Surveillance in a time of changing health care practices:estimating ectopic pregnancy incidence in the United States. Matern Child Health J. 2002;6(4):227-236.

5. Creanga AA, Shapiro-Mendoza CK, Bish CL, et al. Trends in ectopic pregnancy mortality in the United States:1980-2007. Obstet Gynecol. 2011;117(4):837-843.

6. Kulp JL, Barnhart KT. Ectopic pregnancy:diagnosis and management Women's Health (Lond Engl/ Future Medicine) 2008;4:79-87.

7. Alkatout I, Honemeyer U, Strauss A, et al. Clinical diagnosis and treatment of ectopic pregnancy. Obstet Gynecol Surv. 2013;68(8):571581.

8. Feng C, Chen ZY, Zhang J, et al. Clinical utility of serum reproductive hormones for the early diagnosis of ectopic pregnancy in the first trimester. J Obstet Gynaecol Res. 2013;39(2):528-535.

9. Wu G, Yang J, Xu W, et al. Serum beta human chorionic gonadotropin levels on day 12 after in vitro fertilization in predicting final type of clinical pregnancy. J Reprod Med. 2014;59(3-4):161-166.

10. Ugurlu EN, Ozaksit G, Karaer A, et al. The value of vascular endothelial growth factor, pregnancy-associated plasma protein-A, and progesterone for early differentiation of ectopic pregnancies, normal intrauterine pregnancies, and spontaneous miscarriages. Fertil Steril. 2009;91(5):1657-1661.

11. Practice Committee of American Society for Reproductive Medicine. Medical treatment of ectopic pregnancy:a committee opinion. Fertil Steril. 2013;100(3):638-644.

12. Dogra V, Paspulati RM, Bhatt S. First trimester bleeding evaluation Ultrasound Q. 2005;21(2):69-85.

13. Dialani V, Levine D. Ectopic pregnancy:a review. Ultrasound $Q$. 2004;20(3):105-117.

14. Condous G, Okaro E, Khalid A, et al. The accuracy of transvaginal ultrasonography for the diagnosis of ectopic pregnancy prior to surgery. Hum Reprod. 2005;20(5):1404-1409.

15. Doubilet PM, Benson CB, Bourne T, et al. Diagnostic criteria for nonviable pregnancy early in the first trimester. $N$ Engl $\mathrm{J}$ Med 2013;369(15):1443-1451.

16. Ghaneie A, Grajo JR, Derr C, et al. Unusual ectopic pregnancies:sonographic findings and implications for management. $J$ Ultrasound Med. 2015;34(6):951-962.

17. Condous G, Kirk E, Lu C, et al. There is no role for uterine curettage in the contemporary diagnostic workup of women with a pregnancy of unknown location. Hum Reprod. 2006;21(10):2706-2710.

18. Seeber BE, Barnhart KT. Suspected ectopic pregnancy. Obstet Gynecol. 2006;107(2 Pt 1):399-413.

19. Monteagudo A, Minior VK, Stephenson C, et al. Non-surgical management of live ectopic pregnancy with ultrasound-guided local injection:a case series. Ultrasound Obstet Gynecol. 2005;25(3):282288

20. American College of Obstetricians and Gynecologists. ACOG Practice Bulletin No. 94:Medical management of ectopic pregnancy. Obstet Gynecol. 2008;111(6):1479-1485. 
21. Odejinmi FO, Rizzuto MI, Macrae RE, et al. Changing trends in the laparoscopic management of ectopic pregnancy in a London district general hospital:7-years experience. J Obstet Gynaecol. 2008;28(6):614-617.

22. Peterson HB, Xia Z, Hughes JM, et al. The risk of ectopic pregnancy after tubal sterilization. U.S. Collaborative Review of Sterilization Working Group. N Engl J Med. 1997;336(11):762-767.

23. Tarraf W, Vega W, González HM. Emergency department services use among immigrant and non-immigrant groups in the United States. $J$ Immigr Minor Health. 2014;16(4):595-606.

24. Rask KJ, Williams MV, Parker RM, et al. Obstacles predicting lack of a regular provider and delays in seeking care for patients at an urban public hospital. JAMA. 1994;271(24):1931-1933.
25. Kyriacou DN, Handel D, Stein AC, et al. BRIEF REPORT:Factors affecting outpatient follow-up compliance of emergency department patients. J Gen Intern Med. 2005;20(10):938-942.

26. Weissman JS, Stern R, Fielding SL, et al. Delayed Access to Health Care:Risk Factors, Reasons, and Consequences. Ann Intern Med. 1991;114(4):325-331.

27. Batool Naqvi SS. Ectopic pregnancy after bilateral tubal ligation. Pakistan Armed Forces Medical Journal. 2014;64(2):378-379. 